\title{
The Impact of Early Design Phase Risk Identification Biases on Space System Project Performance
}

\author{
John D. Reeves \\ Space Mission Analysis Branch \\ NASA Langley Research Center \\ Hampton, U.S. \\ john.d.reeves@nasa.gov
}

\author{
Dr. Tim Eveleigh, Dr. Thomas Holzer, and Dr. \\ Shahryar Sarkani \\ Department of Engineering Management and Systems \\ Engineering \\ George Washington University \\ Washington, U.S.
}

\begin{abstract}
Risk identification during the early design phases of complex systems is commonly implemented but often fails to result in the identification of events and circumstances that truly challenge project performance. Inefficiencies in cost and schedule estimation are usually held accountable for cost and schedule overruns, but the true root cause is often the realization of programmatic risks. A deeper understanding of frequent risk identification trends and biases pervasive during space system design and development is needed, for it would lead to improved execution of existing identification processes and methods.
\end{abstract}

Keywords-costs; project management; risk analysis; space vehicles; systems engineering and theory

\section{INTRODUCTION}

A commonly stated problem across space system design and development efforts is that severe deficiencies exist within system cost and schedule estimating methodologies. Although such criticism is valid to an extent, the assignment of causality from flawed cost and schedule estimating efforts to subsequent cost and schedule overruns often overlooks deeper seated root causes. Oftentimes, the system design itself, from a mass, power, and performance perspective, has significantly evolved from the initial design used for cost and schedule estimating purposes. One can argue that such growth should have been accounted for by the inclusion of adequate margin within performance, cost, and schedule planning. However, such planning requires some amount of anticipation of the types of challenges that a development project will face during the project life cycle. Risk management, as a sub-discipline to project systems engineering efforts, is typically tasked with anticipating and managing such challenges. If the world was one in which the same set of challenges and threats recur within each development effort, the likelihood and impact of these events could easily be assessed and protected against. Instead, each project is prone to its own set of challenges some foreseeable, some not - that risk managers struggle to anticipate. When unanticipated events do happen of enough impact that they surpass budgeted reserves, they can quickly disrupt well formulated plans to the point of significant cost and schedule growth. For example, a recent NASA development project experienced a slip in launch date due to budget reductions (foreseeable) that resulted in even longer delays when the launch service provider had difficulty in reallocating their launch amidst crowded manifests (arguably unforeseeable) [1]. Because of this, this paper suggests that limitations within risk identification efforts, particularly subjective biases related to likelihood and consequence expectations, can and do lead to significant cost and schedule overruns.

This paper discusses the early findings of a research effort aimed at better understanding the biases that may exist in space system risk identification, and how they appear to impact project performance. The intent is to evaluate temporal trends of risks identified throughout the life cycle of numerous projects to understand how early, or how late, the true challenges facing project performance are identified. The outcome is intended to be statistical confirmation that such biases do exist, along with general heuristics that can be used by future project managers to better avoid under appreciating the types of risks that do make a difference. Building upon existing literature that suggests such biases exist, this research further aims to confirm these behaviors using actual historical project data from a variety of NASA space system projects. These results pertaining to NASA projects, being large scale U.S. Government funded efforts, are estimated to be indicative of complex projects across numerous other industries and sectors. To that end, findings and trends are broadly applicable to a variety of applications.

\section{RISK IDENTIFICATION WITHIN NASA}

Current NASA procedural requirements define risk management as "a set of activities aimed at achieving success by proactively risk-informing the selection of decision alternatives and then managing the implementation risks associated with the selected alternative" [2]. This definition correctly indicates that risk management is an activity that begins at the beginning of a project (e.g., when selecting between alternatives) and continues throughout the life of project. In fact, emphasis is equally placed on early riskinformed design decisions as well as continuous management 
of such risks. Instead of choosing a design alternative and worrying downstream about what risks may or may not pose problems, the various risk implications are viewed as decision criteria and managed thereafter. NASA's procedural documents define this approach as a combination of Risk Informed Decision Making (RIDM) and Continuous Risk Management (CRM) [2]-[4]. RIDM pertains to the early phase decision making aspect while CRM is the implementation task of managing the identified risks, generally over the entire set of project life cycle phases [5]. One tool commonly used to implement the CRM philosophy is the risk matrix methodology.

The NASA Systems Engineering Handbook defines the NASA project standard " $5 \times 5$ " risk matrix methodology, commonly used across the entire space sector, as a way to "combine qualitative and semi-quantitative measures of likelihood with similar measures of consequences" [6]. The strengths are that it is easily understood, can assist in tracking top-ranked risks at a high level, and can be easily used to communicate risk status information both within a project as well as to project stakeholders. However, there are numerous drawbacks - such as the fact that combinatorial interactions amongst multiple risks are not accounted for, aggregate risks are not quantified, uncertainty quantification is lacking, and general oversimplicity. At a high level, the standard risk matrix (Fig. 1) is partitioned into three sections based on the intersection of a risk's estimated likelihood and consequence. Per the NASA Systems Engineering Handbook, a low (green) risk "has little or no potential for increase in cost, disruption of schedule, or degredation of performance" [6]. The moderate/medium (yellow) items "may cause some increase in cost, disruption of schedule, or degredation of performance". Finally, the high (red) risk is "likely to cause significant increase in cost, disruption of schedule, or degredation of performance". Although the matrix provides a useful way to convey how risks fall within the green, yellow, or red sections, as well as how they compare to one another in regards to likelihood and consequence, it falls short in providing context behind each risk. There is no clear way to capture the consequence of multiple risks being realized concurrently (i.e., the combined consequence could be greater than the sum of their individual consequences). Another drawback from the matrix approach is that one could infer that a risk with a likelihood of 2 and consequence of 4 is equal in impact $(\mathrm{L} \times \mathrm{C})$ to one with a likelihood of 4 and consequence of 2 , which may often not be the case.

There are also further deficiencies of the standard $5 \times 5$ risk matrix documented in literature. For example, Cox points out that although their use is wide spread and commonly considered standard practice, there has been very little empirical evidence from studies showing their actual efficacy in managing risks [7]. That work points out several potential mathematical and logic flaws that result from the way risks are discretized and mapped; namely logic consistency, mathematical symmetry, and consistent coloring [7].

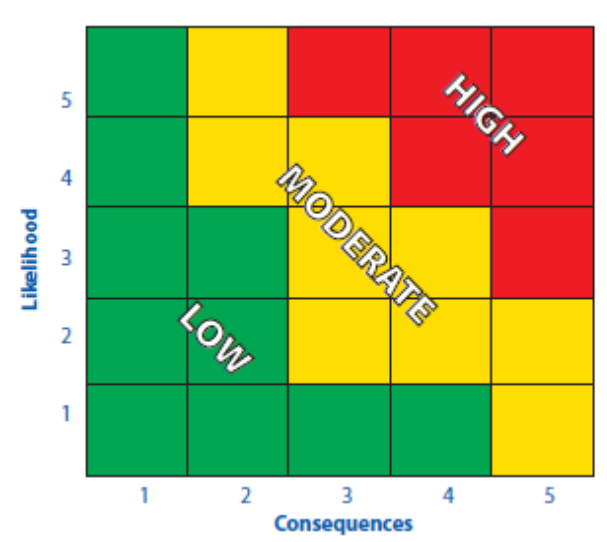

FIGURE 1. NOTIONAL RISK MATRIX [6]

Despite the documented limitations in using standard $5 \times 5$ risk matrices, this research leans heavily on the concept due to its preponderance amongst historical project documentation. For instance, each monthly NASA status document, regardless of which NASA Center is managing the project, routinely includes a $5 \times 5$ risk matrix containing the top 5-10 risks currently threatening the project at that time. Because such documentation is refreshed on a monthly basis it provides a rich data source for understanding temporal trends associated with these top risks. Combined with cost and schedule monthly status documentation, these risks trends provide insight into where biases potentially reside in project risk identification and tracking efforts.

\section{BIASES WITHIN RISK IDENTIFICAITON}

\section{E. Risk Identificaiton Background}

Despite procedural requirements dictating that RIDM and CRM be used to actively identify and track various risks, the actual methodology used to identify the risks is not standardized. Each project typically has either a dedicated risk manager and/or risk management team, and it is up to those persons(s) to decide what methods will be used to identify the litany of possible risks. There have been numerous attempts in research to identify risk identification methodologies that span a variety of approaches such as formalized brainstorming workshops, the use of historical risk registers, and numerous expert elicitation methods. Maytorena et al. [8] provides an indepth literature review of project risk identification methods, and points out the limitations with each of those methods commonly used. That research also explores how risk managers seek and gather information related to potential risks, and demonstrates that reliance on experience and historical risk registers may actually be counter-productive to proper risk identification due to an overreliance on a "check-list mentality" [8]. One reason for such behavior may be continued emphasis on past experience and various challenges that are generally within the control of project and risk managers. Because of this reliance on what has happened in the past, risk managers are prone to focus on the more common, higher likelihood, events. 
Unfortunately, this results in a lack of appreciation for events that, although considered very unlikely to happen, come with more severe consequences.

\section{F. Gray Swans and the Hidden Consequences}

In 2007 Nassim Nicholas Taleb popularized the concept of a "Black Swan" event by describing situations where an event occurs that is unprecedented in nature, thus impossible to some degree to anticipate, but that has catastrophic consequences [9], [10]. The metaphor refers to the fact that in historical European times phrases such as "as rare as a black swan" were used to express an impossibility, for at that point in time black swans were presumed not to exist. After such swans were subsequently discovered during Australian exploration, the phrase evolved to represent events that are thought to be impossible, but whose slim margin of likelihood do allow them to occur. When the associated consequences are severe, those events, despite being considered extremely unlikely to happen, can have far more impact than routine negative events that commonly occur. Although a generalization, the concept has roots in basic statistical inference when a proposed event cannot be confirmed to have zero likelihood probability, even with an infinite amount of observations of non-occurrence [10]. All it takes is a single occurrence to negate the assumption of impossibility. Unfortunately, that single occurrence could take place right when the associated negative consequences would cause the most disruption.

From a project risk management perspective, black swans can and do take place. However, it is an impossibility itself to protect a project against their impact. Fortunately, the occurrence of such an event is inherently unlikely, so in the event something completely unprecedented and unforeseeable occurs the project would just have to proceed as best as possible. From this concept, a middle ground can be perceived - that there are events that are extremely rare and have happened, have significant consequence, but have undeterminable likelihood due to lack of knowledge or experience. Taleb refers to these as "Gray Swans". The unfortunate aspect of these types or risks is that they can potentially be protected against, but are commonly dismissed due to their low likelihood of occurrence. These are the events, based on anecdotal evidence hoped to be confirmed by this research, that end up causing the most disruption to cost and schedule plans. Although their likelihood is low, resulting in managers dismissing them from concern, they do occur often enough to create problems.

The intent of this paper is not to validate the concept of black or gray swans, but to use the latter concept to provide context on the potential biases present in risk identification activities.

\section{G. Perceived Biases}

Similar to many other subjective activities, risk identification is prone to various types of biases related to the understanding of occurrence and consequence. Various literature supports this claim, often by verifying that a large preponderance of risks identified are routine in nature and not representative of the full breadth of events that could take place [10] - [12]. Letens et al. describe this imbalance as a focus on the "Individual Dimension". They documented these as $70 \%$ of the risks studied [11]. This term is defined to include the risks originating from within as opposed to outside. From a project perspective this is interpreted as internal to the project versus external. Kolltveit et al. describe "External" sources of uncertainty separate from "Internal" sources of uncertainty by defining external to include the political situation, contractual variability, and other sources of variability and risk that can impact a project [12]. In contrast to internal sources such as design, internal organization, and internal goals, the external sources are largely outside of the control of the management personnel. The concept of internal vs. external is not just limited to a project itself, for risks may originate from both within the governing Agency itself or from elsewhere. Coonce et al., in an effort to better understand cost and schedule growth, used a root cause taxonomy that delineated events internal and external to NASA as well as internal and external to an individual project [13]. Events that could be external to a project but internal to NASA include events such as funding variability from Headquarters and guideline mandates from field Centers. Similar to the Letens et al. research, it is speculated here that a large preponderance of identified risks within past NASA projects are of the internal to the project nature, followed by external to project but internal to Agency, and then finally by those external to the Agency. However, it may very well be that the risks external to the project are the ones that actually cause disruption to cost and schedule. This dichotomy, along with an investigation of related behaviors stemming from temporal trends in risk identification, is the focus of this research's methodology.

\section{HYPOTHESES AND METHODOLOGY}

\section{E. Hypotheses}

Based upon the literature in risk identification within project management, the author's experience researching cost and schedule growth, and anecdotal observations by various project managers, a set of hypotheses can be established that will be used to guide an understanding of risk identification biases.

The first hypothesis captures the sense that more focus is spent on identifying and worrying about risks that are the most familiar. These are typically the risks internal to a project, for they are based on past experience and can be somewhat mitigated by actions taken be the project team itself. Data analysis across a number of projects, combined with a risk categorization taxonomy, will provide insight into the support of this hypothesis. Demonstrating that this theory is significant would indicate that risks are identified with a bias towards internal concerns. Simply stated, this hypothesis can be phrased as:

\section{H1: Risks identified by a project are more often internal to a project than external.}


The second hypothesis aims to support that the risks that actually end up causing the most disruption are more commonly external than internal. This could be because internal risks are more easily mitigated upon identification, but also could indicate that events outside of the control of the project can and do pose a bigger threat.

\section{H2: The risks that become drivers of cost and schedule change are more often external to the project than internal.}

The combination of $\mathrm{H} 1$ and $\mathrm{H} 2$, if both are supported, would underscore the belief that there is a disconnect between what types of risks are identified versus what risks are the bigger concern. A project may not be able to actively manage a risk external to their project, but an increased awareness would allow that project to better understand who, externally, could potentially mitigate the risk to the betterment of the project situation. To enable such, it is important to gain an understanding into how early a typical project actually identifies these types of events.

The third and fourth hypotheses aim to gain an understanding of the temporal trends associated with identifying external risks. The thought is that these types of events can be identified as early as conceptual design, but are more commonly only identified once they become an active problem. Because of this, they are identified later and tracked for a shorter duration than other risks. The duration measurement is of interest because it is thought that external risks are often caught much closer to risk realization than internal risks. In other words:

\section{H3: Risks external to a project are not identified as early as risks internal to a project.}

\section{H4: Risks external to a project are not tracked as long as risks internal to a project.}

Since hypotheses three and four are specific to only external risks regardless of their actual impact, investigating the temporal behavior of all risks that pose a cost and schedule problem, whether they are internal or external, may be worthwhile:

\section{H5: Risks that become drivers of cost and schedule change, regardless of whether they are internal or external, are not identified as early as other risks.}

\section{F. Data}

The intent of this research is to substantiate anecdotal observations made when reviewing past NASA projects. To that end, actual NASA project data is leveraged in the form of monthly reports provided by field centers to NASA Headquarters in Washington, D.C. Although there is variability from one center to the next, and from one project to the next, there is some consistency in how each project's risk status is documented each month. Among other data, each typically includes a standard $5 \times 5$ risk matrix with the top 5-10 risks identified and discussed. This information, in combination with temporal trends in cost and schedule plans, will provide insight into the hypotheses described above.

NASA is divided into several high-level mission directorates; Human Exploration \& Operations, Aeronautics, and Science. Within the Science Mission Directorate (SMD), there are four divisions based on the purpose of missions contained within; Earth, Heliophysics, Planetary, and Astrophysics. As with all Mission Directorates, SMD is spread amongst a number of centers across the country. Data for this research was pulled from the Jet Propulsion Laboratory (JPL) in Pasadena, California, The Goddard Space Flight Center (GSFC) in Greenbelt, Maryland, and the Langley Research Center (LaRC) in Hampton, Virginia. Within SMD, a centralized database is used at NASA Headquarters to document and store the monthly status reports provided by each of the field centers pertaining to development project status. This research is based on the data contained within that database, with permission having been obtained from the SMD Chief Engineer (representing ownership of the database) as well as key personnel from JPL, GSFC, and LaRC (representing ownership of the projectspecific data).

Data collection is still underway, with significant amount of time required to review each monthly status document to extract risk, cost, and schedule information (also cost, schedule, and mass margin detail in case helpful during future analysis). Based on a review of the available data within the database, thirty different missions have been identified that may have enough documented data to support analysis. Those 30 projects span the centers as well as all four mission divisions within SMD. To date, cost, schedule, and risk data has been gathered from two missions, and constitutes the data set for the preliminary findings contained within this paper.

Because this research aims to identify and verify trends at the high level for wide-spread applicability to other industries and agencies, published results are kept at an aggregate level without specificity to any one project or center.

\section{G. Research Framework}

The methodology used will be a quantitative statistical evaluation of the various hypotheses discussed. To frame the analysis, a research framework has been developed and can be seen in Fig. 2. Within the research framework diagram there are four sets of variables defined. The "Risks Identified" represents the data gleaned from various projects across NASA SMD divisions and centers. These data include the top-level risks identified each month, along with monthly status measurements of project cost and schedule progress. Based on these data, when evaluated over time, temporal trends can be discerned which are accounted for in the "Temporal Trends" variable seen below. 


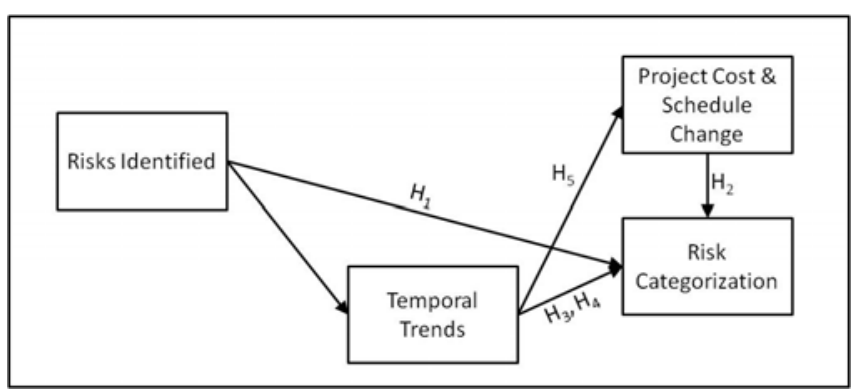

FIGURE 2. RESEARCH FRAMEWORK

The first portion of analysis includes the assignment of a risk category specification to each risk. This assignment is based on the author's interpretation of documentation associated with each project risk, and is based on a taxonomy described in a subsequent section. These categorization results, seen in the framework as "Risk Categorization", represent dependent variables due to their dependability on the "influence of the independent variables" [14], although in this particular circumstance are also somewhat based on the author's subjective interpretation. The final set of variables, "Project Cost \& Schedule Change", represents cost and schedule measurements pulled from the data sets. Although independent in the sense they are raw project data, they are considered dependent here relative to the risks and risk trends identified.

\section{H. Cost and Schedule Change Variables}

To understand which risks have correlation to cost and schedule change, the various cost and schedule measurements from the data sources are used. In each case a baseline cost and schedule can be gleaned from early project documentation, and can be used as a reference in which to gauge subsequent growth in either measurement. The primary cost measurement is the project's total life cycle cost, which includes costs from Phase A through Phase E as defined in the NASA Systems Engineering Handbook [6]. A growth percentage is used to account for percent change from the baseline to subsequent levels. The schedule measurement is broken down into three milestone components; Preliminary Design Review (PDR), Critical Design Review (CDR), and Launch Readiness Data (LRD)/Launch. The latter milestone is considered readiness date during early design phases but becomes an actual launch milestone upon successfully mission launch. To quantify schedule growth a "months slipped" measurement will be tracked for each of the three schedule measurements.

It is thought that the cost growth measurement will be more commonly indicative of cost and schedule growth since a number of missions, particularly planetary missions, have fixed launch windows due to mission opportunity windows related to orbital mechanics. However, both the cost and schedule growth measurements will be used as dependent variables within the research framework.

\section{Risk Characterization Taxonomy}

Within the literature there are numerous taxonomies used to understand the differences in risk and types of uncertainty [12], [13], [15]-[17]. Despite whether research is based on space systems, software development, or other industries, there seems to be a wide-spread delineation within the taxonomies between internal and external sources. Because of this, having any taxonomy used here include emphasis on such is important. Choosing a taxonomy that is at an appropriate level to support the data used within this research is also important, for the data is of a level typically reported to NASA Headquarters, as opposed to detailed case-study level research that could be garnered from an extensive review of a single project or two. Based on these needs, a taxonomy was chosen based on similar research performed by NASA Headquarters and the Aerospace Corporation [13]. That work focused on the characterization of root causes amongst past NASA projects, but with some small modifications the same taxonomy can be used here. Table 1 below captures the modified taxonomy used to label each of the risks identified to date.

TABLE 1. RISK CHARACTERIZATION TAXONOMY

\begin{tabular}{|c|c|c|c|c|}
\hline ID & Agency & Project & Area & Sub-Area \\
\hline PP1 & NASA Internal & Project Internal & Project Planning & Design - Spacecraft \\
\hline PP2 & NASA Internal & Project Internal & Project Planning & Design - Instrument \\
\hline PP3 & NASA Internal & Project Internal & Project Planning & Technology-Readiness \\
\hline PP4 & NASA Internal & Project Internal & Project Planning & Technology - Contingency/Backup \\
\hline PP5 & NASA Internal & Project Internal & Project Planning & Cost and Schedule Plans \\
\hline PP6 & NASA Internal & Project Internal & Project Planning & Programmatic \\
\hline PP7 & NASA Internal & Project Internal & Project Planning & Heritage \& Commonality \\
\hline PP8 & NASA Internal & Project Internal & Project Planning & Other \\
\hline PE1 & NASA Internal & Project Internal & Project Execution & Management - PM/SE/MA Growth \\
\hline PE2 & NASA Internal & Project Internal & Project Execution & Management - Requirements Growth \\
\hline PE3 & NASA Internal & Project Internal & Project Execution & Sys Development - Spacecraft \\
\hline PE4 & NASA Internal & Project Internal & Project Execution & Sys Development - Instrument \\
\hline PE5 & NASA Internal & Project Internal & Project Execution & Sys Development - Ingration \& Test \\
\hline PE6 & NASA Internal & Project Internal & Project Execution & Sts Developement - Ground Systems \\
\hline PE7 & NASA Internal & Project Internal & Project Execution & Contractor Management \\
\hline PE8 & NASA Internal & Project Internal & Project Execution & In-House Performance \\
\hline PE9 & NASA Internal & Project Internal & Project Execution & Other \\
\hline AL1 & NASA Internal & Project External & Agency Level & Annual Funding \\
\hline AL2 & NASA Internal & Project External & Agency Level & Program Requirements \\
\hline AL3 & NASA Internal & Project External & Agency Level & Component Supplier \\
\hline AL4 & NASA Internal & Project External & Agency Level & Forced Budget Cap \\
\hline AL5 & NASA Internal & Project External & Agency Level & Accounting Changes \\
\hline AL6 & NASA Internal & Project External & Agency Level & Other \\
\hline CL1 & NASA Internal & Project External & Center Level & Principles or Guidelines \\
\hline $\mathrm{CL2}$ & NASA Internal & Project External & Center Level & Workforce \\
\hline CL3 & NASA Internal & Project External & Center Level & Estimating Process \\
\hline CL4 & NASA Internal & Project External & Center Level & Center Facilities \\
\hline CL5 & NASA Internal & Project External & Center Level & Other \\
\hline NE1 & NASA External & N/A & N/A & Launch Vehicle \\
\hline NE2 & NASA External & N/A & N/A & Congress/OMB \\
\hline NE3 & NASA External & $\mathrm{N} / \mathrm{A}$ & N/A & Force of Nature \\
\hline NE4 & NASA External & N/A & N/A & Industrical Base Issue \\
\hline NE5 & NASA External & N/A & N/A & Economic Issues \\
\hline NE6 & NASA External & N/A & N/A & Partner Performance \\
\hline NE7 & NASA External & $\mathrm{N} / \mathrm{A}$ & N/A & Other \\
\hline
\end{tabular}

As can be seen, there are several layers of delineation amongst the categories. First, there is a separation between risks internal to NASA and external to NASA. Risks internal to NASA include items related to project performance, development challenges, Agency level mandates, and so forth. Risks external to NASA include items such as launch vehicle delays (since NASA often contracts launch service providers that are prone to their own set of schedule conflicts), forces of nature, and nationwide economic variability.

Within the NASA Internal set of risks, there is a separation between risks internal and external to a project. Internal project risks typically relate to project planning and performance, while external project risks include agency and center level 
requirements and implications. Within Project Internal the risks are separated into Project Planning and Project Execution. Within Project External the risks are separated into agency and center level risks.

Because the original taxonomy used by the Aerospace Corporation [13] was specific to root cause analysis, several changes were made to adopt it to this research. First, a Project Planning item related to risk identification was changed to "Cost and Schedule Plans". Secondly, within that same section a "Heritage and Commonality" risk category was added to capture events where common hardware from other missions poses an issue. Within the Project Execution area a "Risk Mitigation" area was changed to "In-House Performance" to capture internal project risks related to underperforming inhouse efforts. Within the Center Specific set of categories a "System Development" item was changed to "Center Facilities" to capture risks where center test facilities caused delays. Finally, a NASA External risk was added to capture partner performance.

\section{DATA ANALYSIS AND RESULTS}

Since the intent of this paper is to describe the longer term methodology while including only some preliminary results, only analysis relating to hypotheses 1, 3, and 4 are discussed herein. After data collection from an increasing number of projects is completed, along with thorough review of each to understand causes of cost and schedule change, analyses relating to hypotheses 2 and 5 will begin.

Between the two projects studied a total of 135 risks were identified and characterized per the taxonomy mentioned above. The split between the two projects was roughly even, with one project providing $56 \%$ of the risks while the other $44 \%$. Along with the assigned categorization of each risk, additional data in the forms of the starting month of each risk, the duration in months when each risk was tracked, as well as the average risk rank, likelihood value, and consequence value across those months was gathered.

\section{E. Hypothesis 1}

A first level understanding of $\mathrm{H} 1$ (risks identified by a project are more often internal to a project than external) can be gained by a comparison of the number of external versus internal risks across the 135 data points. The results can be seen in Fig. 3, and are indicative that an overwhelmingly majority $(116,86 \%)$ of the risks are considered internal to the project. Outside of the project there are more Internal to NASA $(15,11 \%)$ than External to NASA (4, 3\%). From this observation alone, based on this initial data set, it is clear that $\mathrm{H} 1$ is supported.

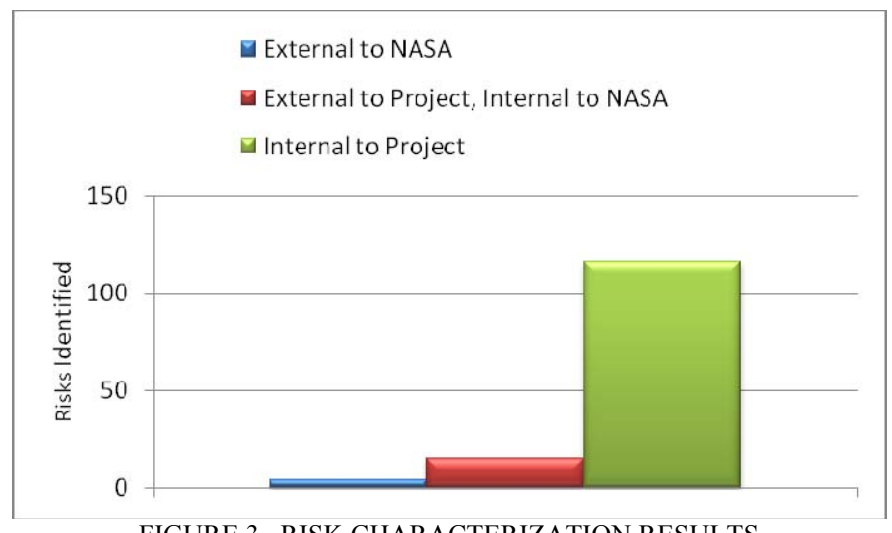

FIGURE 3. RISK CHARACTERIZATION RESULTS

\section{F. Hypothesis 3}

To investigate H3 (risks external to a project are not identified as early as risks internal to a project), a comparison is made of the average month, within a project's life cycle, in which each of the types of risks were initially identified. Since different projects have different length development durations, and took place over a different set of years, the data was normalized such that a point in time was represented as a percentage between when risk tracking began and launch. This normalization allows a valid comparison of the points in time in which risks were initially identified during the development activity.

Although statistical tests were planned to validate statistical significance of $\mathrm{H} 3$, from observations of the data the hypothesis not being supported became clear. For risks internal to projects, the mean value, as a percentage, of when they were identified during the project was $42 \%$. For risks external to projects this same value was $39 \%$, thus on average taking place earlier than the internal risks.

\section{G. Hypothesis 4}

To understand if $\mathrm{H} 4$ (risks external to a project are not tracked as long as risks internal to a project) can be supported, the average duration, in months, of each type of risk was evaluated. From a quick comparison, one can see that the mean duration of tracking for external projects (2.79 months) was shorter than internal projects $(2.84)$. To check for statistical significance a $\mathrm{Z}$ test comparing two sample means with variance known was applied.

In this case, the null hypothesis was assumed to be that internal project risk duration was less than or equal to external duration. By statistically rejecting the null, H4 could have been demonstrated. However, when assuming alpha values of either $\alpha=0.05$ or $\alpha=0.1$ the test value does not fall within the critical range, thus there is not enough evidence to reject the null. Although the mean value for external risks is smaller than that of internal risks, the difference is not enough to be statistically significant. 


\section{H. Additional Analysis}

Using the same data collected and analyzed, statistical comparisons were also made to determine if the average likelihood or consequence values were greater for internal risks than external risks. This behavior would be indicative of a bias to consider the internal threats both more likely to happen as well as have the potential for greater impact to the project.

In both cases the calculated mean values appeared to support the claim, with a mean value of 2.84 for likelihood and 3.54 for consequence for internal risks, compared to a mean value of 2.79 for likelihood and 3.47 for consequence for external risks. However, when the same $\mathrm{Z}$ test was applied in each case there was not enough evidence to support the observations.

\section{Observations}

Based on these preliminary findings, the expected biases may exist within the data, but that additional data collection and analysis is warranted. Although H1 was clearly demonstrated based on observation, neither $\mathrm{H} 3$ nor $\mathrm{H} 4$ were supported. H1 confirmed that a large preponderance of risks identified were internal in nature, with only a small percentage being either external to the project or external to NASA altogether. This is consistent with literature suggestions that risk identification efforts do not always fully incorporate both internal and external concerns.

Hypothesis 3 was not supported based on the observation that the mean identification month for external projects was not later than that for internal projects. This is inconsistent with expected results, but could be due to the smaller set of data currently being evaluated.

Hypothesis 4 appeared to be supported based on initial observation, but was not shown to be significant. The same is said for additional analyses that evaluated the average likelihood and consequence ratings for each risk. Additional planned analysis using extended data sets may ultimately provide statistical significance in these areas.

Hypotheses 2 and 5 will be evaluated in future analyses using additional review of current data as well as the incorporation of additional data sets from a greater number of NASA projects.

\section{CONCLUSION AND BROADER APPLICABILITY}

Risk management methodologies are commonly used throughout government space system design as part of systems engineering efforts to identify, track, and manage the various challenges and threats that each project faces. An underlying tenet to this research activity is that the realization of certain types of risks, and the biases that prevent them from being fully anticipated, causes just as much disruption to cost and schedule plans than other commonly stated root causes. By demonstrating that these biases, often relating to risks external to a project manager's control, do exist and are impactful, future project and risk managers will be more prepared to acknowledge these biases.
The research framework and methodology described herein is being formulated to guide a year-long investigation using extensive NASA historical project data. Thirty projects have been identified across three NASA Centers and all four Science Mission Directorate Divisions that will be potentially mined for supporting risk, cost, and schedule data. The result of this data collection and subsequent analysis will be thorough investigation into the biases speculated to exist.

To date two of the thirty projects have been evaluated, providing 135 top-level risks that were of importance enough to be contained in monthly $5 \times 5$ risk status matrices. The results contained herein, with analysis findings documented related to hypotheses 1, 3 and 4, are based on these 135 data points. As additional data is collected and analyzed it is expected that these preliminary findings will evolve. In addition, subsequent causality analysis of risk realization to cost and schedule change will be incorporated, allowing thorough statistical evaluation of Hypotheses 2 and 5.

Although data evaluated is NASA SMD data, biases verified within these activities are believed to be indicative of biases that exist throughout all complex large-system development efforts.

\section{ACKNOWLEDGEMENT}

The authors would like to thank personnel at NASA that have approved the use of NASA project data for the purposes of this research. Those individuals include Ken Ledbetter at NASA Headquarters, Chris Jones at NASA JPL, Dr. Lelia Vann at NASA Langley, and Steve Shinn at NASA Goddard.

Thanks is also given to Bob Bitten at the Aerospace Corporation for helping the authors better understand the taxonomy used in reference [13] that was the basis for risk characterization within this research.

\section{REFERENCES}

[1] GAO, "Assessments of Selected Large-Scale Projects (GAO-10-227SP)," United States Government Accountability Office, 2010.

[2] NASA, "NPR: 8000.4A - Agency risk management procedural requirements," Office of Safety and Mission Assurance, 2008.

[3] NASA, "NASA Risk-Informed Decision Making Handbook," NASA, Washington, 2010.

[4] H. Dezfuli, G. Maggio and C. Everett, "Risk-informed decision making: Application to technology development alternative selection," in 4th IAASS Conference, Huntsville, 2010.

[5] E. A. Silk and P. H. Dash, "Risk management in space systems design and technology development," Journal of Aerospace Engineering, vol. 222, no. 6, pp. 907-913, 2008.

[6] NASA, "NASA Systems Engineering Handbook (SP-2007-6105)," NASA Headquarters, Washington, 2007.

[7] L. A. Cox Jr., "What's wrong with risk matrices?," Risk Analysis, vol. 28, no. 2, pp. 497-512, 2008.

[8] E. Maytorena, G. Winch, J. Freeman and T. Kiely, "The influence of experience and information search styles on project risk identification performance," IEEE Transactions on Engineering Management, vol. 54, 
no. 2, pp. 315-326, 2007.

[9] N. N. Taleb, The Black Swan, New York: Random House, 2007.

[10] A. M. Nafday, "Strategies for managing the consequences of black swan events," Leadership and Management in Engineering, vol. 9, no. 4, pp. 191-197, 2009.

[11] G. Letens, L. V. Nuffel, A. Henne and J. Leysen, "Towards a balanced approach in risk identification," Engineering Management Journal, vol. 20, no. 3, pp. 3-9, 2008.

[12] B. J. Kolltveit, J. T. Karlsen and K. Gronhaug, "Exploiting opportunities in uncertainty during the early project phase," Journal of Management in Engineering, vol. 20, no. 4, pp. 134-140, 2004.

[13] T. Coonce, B. Bitten and B. Kellogg, "Explanation of change (EOC) cost \& schedule growth study - Overview \& interim results," in 2010 International SSCAG/SCAF/EACEWG Joint Meeting, Berlin, 2010.

[14] J. W. Creswell, Research Design: Qualitative, Quantitative, and Mixed Method Approaches, Thousand Oaks, CA: SAGE Publications, 2009.

[15] G. H. Neilson, C. O. Gruber, J. H. Harris, D. J. Rej, R. T. Simmons and R. L. Strykowsky, "Lessons learned in risk management on NCSX," IEEE Transactions on Plasma Science, vol. 38, no. 3, pp. 320-327, 2010.

[16] L. Wallace, M. Keil and A. Rai, "How software project risks affects project performance: An investigation of the dimensions of risk and an exploratory model," Decision Sciences, vol. 35, no. 2, pp. 289-321, 2004.

[17] T. M. Skelton and H. J. Thamhain, "A stakeholder approach to minimizing risks in complex projects," in PICMET 2006 Proceedings, Istanbul, 2006. 